\title{
DESENVOLVIMENTO DE AMOSTRADOR PASSIVO SENSÍVEL PARA MONITORAMENTO DE POLUIÇÃO ATMOSFÉRICA POR DIÓXIDO DE NITROGÊNIO
}

Pedro A. F. Souza, Karen C. A. Francisco e Arnaldo A. Cardoso*

Departamento de Química Analítica, Instituto de Química, Universidade Estadual Paulista, 14800-060 Araraquara - SP, Brasil

Recebido em 04/04/2017; aceito em 31/07/2017; publicado na web em 12/09/2017

\begin{abstract}
DEVELOPMENT OF SENSITIVE PASSIVE SAMPLER FOR NITROGEN DIOXIDE AIR POLLUTION MONITORING. A passive sampler constructed with a new type of material and used for measurement of ambient nitrogen dioxide is presented. The sampler was constructed with parts of two $50 \mathrm{~mL}$ conical centrifuge tubes. The procedure is based on $\mathrm{NO}_{2}$ uptaken on cellulose filter impregnated with triethanolamine. After sampling, the analyte is solubilized and placed to react with Griess-Saltzman solution. The absorbance of the formed complex is determined at $540 \mathrm{~nm}$. A mean relative standard deviation of $9,72 \%$ was obtained using samplers exposed in triplicate, and a determination coefficient $\left(\mathrm{R}^{2}\right)$ of 0,979 was achieved in parallel measurements using the samplers and a commercial NOx monitor. Advantages of the technique include ease of use, the ready availability of components, and excellent sensitivity.
\end{abstract}

Keywords: passive sampler; nitrogen dioxide; air pollution monitoring; air quality.

\section{INTRODUÇÃO}

A avaliação da concentração de gases reativos em ambientes abertos e fechados tem sido um problema analítico frequente no mundo moderno. Gases como o ozônio, dióxido de enxofre e dióxido de nitrogênio podem afetar a saúde humana, ${ }^{1}$ danificar a vegetação natural ${ }^{2-4}$ e degradar objetos que apresentam relevância cultural ${ }^{3,4}$ ou comercial. ${ }^{4,5}$ Quando há amônia na atmosfera em conjunto com gases $\mathrm{SO}_{2}$ e $\mathrm{NO}_{2}$ pode também ocorrer formação de aerossol respirável. ${ }^{6} \mathrm{O}$ gás dióxido de nitrogênio está presente na atmosfera de ambientes abertos e fechados ao redor do planeta. Em ambientes fechados, sua concentração pode alcançar valores que causam efeitos deletérios à saúde humana. ${ }^{7,8} \mathrm{Em}$ ambiente aberto, esse gás, com raríssimas exceções, não atinge concentrações que possam provocar prejuízos à saúde. No entanto, seu controle é necessário uma vez que atua como fotocatalisador na produção do ozônio, ${ }^{2,4,5}$ na formação do ácido nítrico atmosférico ${ }^{9}$ e de aerossol atmosférico e pode interferir no ciclo biogeoquímico de macronutrientes. ${ }^{10,11} \mathrm{O} \mathrm{NO}_{2}$ é um poluente secundário formado na própria atmosfera a partir de reações de óxido nítrico com ozônio (Equação 1) ou com compostos formados em reações fotoquímicas (Equação 2). Processos de combustão utilizados para geração de energia com objetivos diversos, como produzir energia elétrica, movimentar motores industriais e veiculares e para cozinhar alimentos, são as principais fontes antrópicas de emissão do NO para atmosfera. ${ }^{5}$

$$
\begin{aligned}
\mathrm{NO}+\mathrm{O}_{3} & \rightarrow \mathrm{NO}_{2}+\mathrm{O}_{2} \\
\mathrm{HO}_{2}+\mathrm{NO} & \rightarrow \mathrm{NO}_{2}+\mathrm{HO} \\
\mathrm{RO}_{2}+\mathrm{NO} & \rightarrow \mathrm{NO}_{2}+\mathrm{RO}
\end{aligned}
$$

Os problemas que requerem a determinação da concentração de gases nem sempre exigem medidas em tempo real, mas a concentração média diária ou mesmo semanal, e demandam reconhecer quais as regiões do local a ser analisado que são mais afetadas pela concentração de um gás poluente. A média de concentração de um gás ao longo do espaço de horas pode ser convenientemente obtida com o uso de amostradores passivos. Este tipo de amostrador foi inicialmente proposto para ser utilizado em monitoramento de ambientes de trabalho ${ }^{12}$

*e-mail: acardoso@iq.unesp.br e, posteriormente, para monitoramento interno de museus ${ }^{13}$ e outros ambientes fechados. ${ }^{14} \mathrm{~A}$ praticidade dos amostradores passivos, por ser este um objeto de fácil operação e não necessitar de energia elétrica, ampliou sua utilização para ambientes externos ${ }^{3,15,16}$ e o tornou um recurso frequente para a caracterização de amplas regiões com relação a diferentes tipos de poluentes gasosos. ${ }^{17}$

$\mathrm{O}$ amostrador passivo é, usualmente, um objeto cilíndrico de dimensões geométricas conhecidas com uma abertura que permite a entrada do ar contendo o analito de interesse. No interior do amostrador, é criada uma região onde o ar permanece em estado próximo ao estacionário e por onde ocorre a difusão do gás para a superfície de coleta do analito. Na parte superior do amostrador é colocada uma superfície ativa (superfície absorvente), que consiste de um material impregnado com uma solução capaz de reagir eficientemente com o analito de interesse, resultando em concentração zero do analito na interface (Figura 1). Um problema bastante comum de amostradores passivos é a perturbação do ar em estado estacionário dentro do amostrador por ventos ou correntes de ar. ${ }^{18,19}$ É possível minimizar esse efeito colocando uma membrana porosa próxima à abertura do amostrador. Essa barreira física diminui o efeito do vento sobre o ar estagnado em seu interior e, desta forma, possibilita que se trabalhe com um amostrador com menor caminho de difusão, sem que ocorram erros significativos na medida causados pela turbulência do ar. ${ }^{4,18}$

O processo de difusão de um gás pelo interior do amostrador e a determinação da sua concentração na superfície coletora é descrito pela Equação 3, derivada da primeira lei da difusão de Fick: ${ }^{14}$

$$
\mathrm{n}_{\mathrm{x}}=\frac{-\mathrm{D}_{\mathrm{x}}\left(\mathrm{C}_{0, \mathrm{x}}-\mathrm{C}_{\mathrm{x}}\right) \pi \mathrm{r}^{2} \mathrm{t}}{\mathrm{z}}
$$

em que $\mathrm{n}_{\mathrm{x}}$ é a quantidade de analito (mol) recolhida durante o tempo $\mathrm{t}$ de amostragem (segundos), $\pi r^{2}$ é a área transversal do tubo do amostrador $\left(\mathrm{cm}^{2}\right), \mathrm{D}_{\mathrm{x}}$ o coeficiente de difusão molecular do gás $\mathrm{x}\left(\mathrm{cm}^{2} \mathrm{~s}^{-1}\right)$, $\mathrm{z}$ o comprimento do caminho de difusão do gás $(\mathrm{cm})$, medido entre a membrana porosa colocada no amostrador e a superfície absorvente, $\mathrm{C}_{0, \mathrm{x}}$ é a concentração de gás $\mathrm{x}$ na interface da superfície absorvente $\left(\mathrm{mol} \mathrm{cm}{ }^{-3}\right), \mathrm{e} \mathrm{C}_{\mathrm{x}}$ a concentração de gás no ambiente $\left(\mathrm{mol} \mathrm{cm} \mathrm{cm}^{-3}\right) . \mathrm{Em}$ uma superfície absorvente ideal todo o gás $\mathrm{x}$ é coletado e $\mathrm{C}_{0, \mathrm{x}}$ pode ser considerado igual a zero. Quando se determina $\mathrm{NO}_{2} \mathrm{o}$ valor do coeficiente de difusão molecular é tabelado em $0,1361 \mathrm{~cm}^{2} \mathrm{~s}^{-1},{ }^{20}$ 


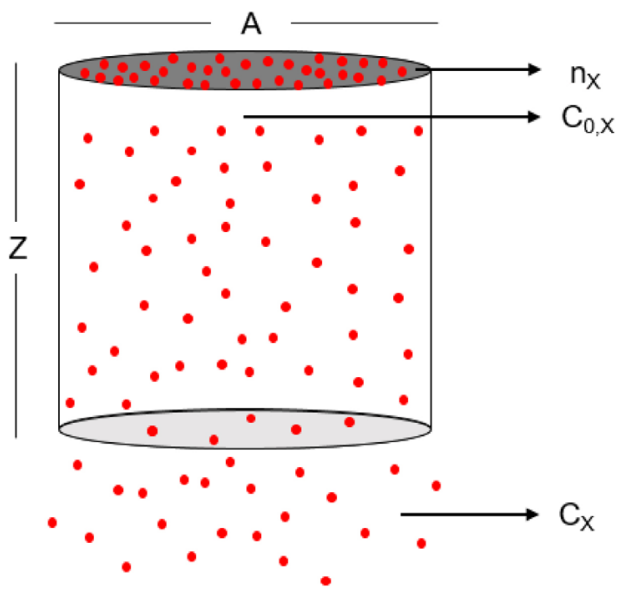

Figura 1. Esquema de um amostrador passivo amostrando um gás $x$. $C_{X}$ é a concentração de gás no ambiente, $C_{0, X}$ é a concentração do gás x na interface da superfície ativa, $n_{X}$ é a quantidade do gás coletado sobre a superfície ativa, A é a área da superfície ativa e $Z$ é o caminho de difusão do gás

ainda que fatores como temperatura e pressão possam ter efeito sobre esse valor. Trabalhos experimentais demonstraram que as alterações causadas por esses parâmetros podem ser negligenciadas. ${ }^{21}$

Apesar da praticidade relacionada à utilização dos amostradores passivos, a compra dos amostradores disponíveis comercialmente ainda é um inconveniente, pois não são encontrados no mercado nacional e sua aquisição requer passar por todos os trâmites legais e morosos dos processos de importação. Opções a estes materiais importados podem ser convenientes não só pelo aspecto econômico, mas também quando se busca uma resposta imediata para a solução de um problema. Neste artigo é apresentada uma proposta de construção de amostrador passivo com materiais de uso comum em laboratório e sua utilização foi avaliada para monitoramento de gás dióxido de nitrogênio em ambientes externos. Os resultados foram comparados com os dados de concentração obtidos por um amostrador ativo comercial de monitoramento contínuo utilizado para controle de poluentes atmosféricos pela Companhia Ambiental do Estado de São Paulo (CETESB).

\section{PARTE EXPERIMENTAL}

\section{Confecção do amostrador passivo}

Para a construção de cada amostrador passivo, foram utilizados dois tubos cônicos Falcon de 50 mL. Tubos cônicos tipo Falcon são recipientes de plástico polipropileno (PP) descartáveis utilizados com frequência para armazenamento e transporte de material químico ou biológico. O custo médio de cada tubo é de cerca de R $\$ 0,70$ ou US\$ 0,20 (2017). Cada tubo cônico foi cortado na marcação de $50 \mathrm{~mL}$, à 1,2 cm abaixo da borda, com o auxílio de uma serra acoplada a uma mini furadeira elétrica. As superfícies das peças cortadas foram lixadas para corrigir algumas irregularidades.

A parte cortada e lixada de uma das peças foi vedada com uma fita de Teflon, vendida no comércio para ser utilizada em conexões com rosca de ligações de água e conhecida como fita veda rosca. Esta serviu como membrana porosa para formar a barreira física que cria a região de difusão e estagnação do ar no interior do amostrador (Figura 2). A fita de Teflon adere facilmente as bordas do tubo sem a necessidade de cola e sem o risco de danificação. O Teflon é um material bastante inerte e dificilmente retêm compostos em sua superfície, portanto, não houve necessidade de tratamento prévio desta fita, uma vez que sua função é de apenas permitir a difusão dos gases para o interior do amostrador e barrar a entrada de material particulado. A parte cortada e lixada de uma outra peça foi colocada sobre a barreira criada com a fita de Teflon e a junção das duas peças foi recoberta com uma fita isolante preta, que é usualmente utilizada para proteger e isolar conexões elétricas, para formar o corpo do amostrador. Além de juntar as duas peças, a fita isolante tem como função evitar ou reduzir a influência da luz solar no interior do amostrador, uma vez que reações fotoquímicas decompõe o $\mathrm{NO}_{2}$ em $\mathrm{NO}$ e oxigênio molecular (Equação 4). ${ }^{22}$ Essa fita é resistente o suficiente para não danificar quando o amostrador é aberto e também não necessita de um tratamento prévio, pois recobre apenas a parte externa do amostrador.

$$
\mathrm{NO}_{2}+h \mathrm{v} \rightarrow \mathrm{NO}+\mathrm{O}
$$

Para manter o amostrador fechado até chegar ao local de amostragem e impedir a entrada do ar atmosférico durante seu transporte, foram utilizadas duas tampas de tubos cônicos tipo Falcon (Figura 2). Os amostradores confeccionados possuíam o caminho de difusão (z) igual a 1,2 cm e o raio (r) igual a 1,3 cm. Essa relação proporciona um caminho de difusão pequeno em relação à área da seção transversal e possibilita um aumento da quantidade de gás coletado $\left(n_{x}\right)$ e, portanto, amostragens em tempos menores. ${ }^{23} \mathrm{O}$ amostrador, quando fechado, tem uma altura total de $2,4 \mathrm{~cm}$ e essa pequena dimensão facilita seu armazenamento e transporte.

Como superfície absortiva, ou seja, meio sorvente para o $\mathrm{NO}_{2}$, foi utilizado filtro de celulose Whatman 41 recortado com o mesmo diâmetro do amostrador e impregnado com solução de trietanolamina $11 \%(\mathrm{v} / \mathrm{v}) .^{14}$

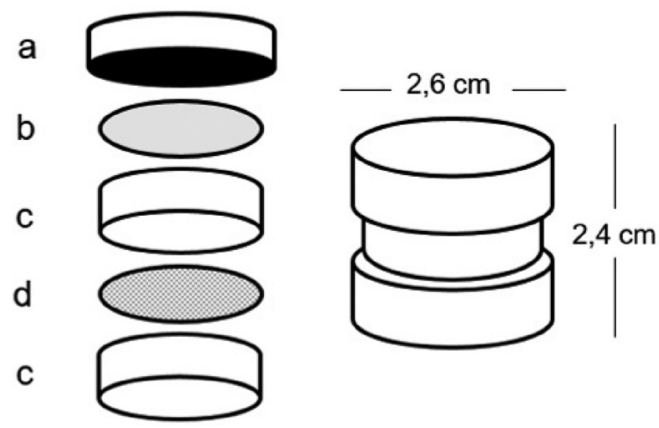

Figura 2. Esquema de montagem do amostrador passivo confeccionado, sendo a a tampa, b a superfície de absorção, c o corpo do amostrador e d a membrana porosa

\section{Reagentes e soluções utilizados}

Todas as soluções aquosas foram preparadas com água purificada por um sistema Milli-Q (18 M $\Omega \mathrm{cm})$ (Direct-Q ${ }^{\circledR} \mathrm{UV}$, Millipore, USA).

Solução de trietanolamina (TEA) absorvente de $\mathrm{NO}_{2}$

Uma solução de TEA $11 \%$ (v/v) foi preparada com a adição de 5,5 mL de trietanolamina (Sigma-Aldrich), 1,75 mL de etilenoglicol (Sigma-Aldrich) e 12,5 mL de acetona (Sigma-Aldrich) à um béquer. Foi adicionada água purificada até volume final de $50 \mathrm{~mL}$. A solução foi guardada em frasco âmbar e armazenada no refrigerador.

\section{Reagente de Griess-Saltzman}

O regente de Griess-Saltzman foi preparado com a adição de 2,50 g de ácido sulfanílico (Sigma-Aldrich) e $70 \mathrm{~mL}$ de ácido acético glacial (Sigma-Aldrich) a um béquer e a mistura diluída com 400 $\mathrm{mL}$ de água purificada. A dissolução do ácido sulfanílico foi facilitada com aquecimento e agitação da solução com uma bagueta de vidro. Após a dissolução completa do sólido, $10 \mathrm{~mL}$ de solução de 
$\mathrm{N}$-(1-naftil)-etilenodiamina $0,1 \%$ foram adicionados e o volume de $500 \mathrm{~mL}$ foi completado com água purificada. A solução foi armazenada em frasco âmbar e estocada no refrigerador. Para utilização é conveniente que a solução reagente esteja em temperatura ambiente. A solução estoque de $\mathrm{N}$-(1-naftil)-etilenodiaminadiidrocloreto $(0,1 \%)$ (Sigma-Aldrich) foi preparada por dissolução de $0,1 \mathrm{~g}$ desse reagente em $100 \mathrm{~mL}$ de água purificada e armazenada em frasco âmbar no refrigerador.

Uma versão desse reagente colorimétrico está disponível comercialmente para ser utilizada na avaliação de presença de nitrito em aquários de água doce e salgada, porém, para que seja utilizado nas análises em laboratório, ele deve passar por todos os procedimentos de calibração usualmente realizados com soluções de uso analítico.

\section{Solução estoque e de trabalho de nitrito de sódio}

A solução estoque de nitrito foi preparada por dissolução de $0,051 \mathrm{~g}$ de $\mathrm{NaNO}_{2}$ (Sigma-Aldrich) em $25 \mathrm{~mL}$ de água purificada. Esta solução pode ser guardada em frasco âmbar por até um ano. ${ }^{14}$ Para a solução trabalho, tomou-se $0,100 \mathrm{~mL}$ da solução estoque e diluiu-os com água purificada até volume final de $10 \mathrm{~mL}$, chegando-se a uma solução de concentração igual a $2,91 \times 10^{-4} \mathrm{~mol} \mathrm{~L}^{-1}$ de nitrito. Esta solução deve ser preparada na hora em que for utilizada.

\section{Montagem dos amostradores e determinação do dióxido de nitrogênio}

Para a montagem dos amostradores, os filtros de celulose foram cortados com diâmetros de $2,6 \mathrm{~cm}$, com o auxílio de tesouras e utilizando-se luvas, para evitar que houvesse contato das mãos do operador com os filtros e, assim, reduzir os riscos de contaminação. Os filtros foram então impregnados com volumes de $80 \mu \mathrm{L}$ da solução absorvente, composta majoritariamente de TEA $11 \%$ (v/v), com o auxílio de pipetas automáticas. Em seguida, utilizando uma pinça, cada filtro foi colocado ainda úmido no interior de uma das tampas e a ela foi fixado o corpo do amostrador, tomando cuidado para que os filtros não ficassem soltos. Uma outra tampa foi colocada para vedar a entrada de ar para o interior do amostrador. $\mathrm{O}$ amostrador preparado foi embalado em saco de polietileno e armazenado em geladeira até o momento de uso.

No local de amostragem, os amostradores foram retirados da embalagem e fixados em um suporte feito de material isopor, com a entrada de ar voltada para o chão para evitar a deposição de poeira sobre a membrana de Teflon durante a amostragem. Este suporte apresentava geometria similar a uma placa espessa, com espaço suficiente para que quatro furos pudessem ser feitos lado a lado e os amostradores encaixados. $\mathrm{O}$ suporte encontrava-se a aproximadamente 1,5 metros do solo, altura que se mostrou adequada para amostragem, e foi fixado em um poste com o auxílio de arames.

Para cada amostragem, um conjunto de 4 amostradores foi fixado no suporte. Após fixados, a amostragem se iniciou quando as tampas que fecham a entrada de ar dos amostradores foram retiradas ao mesmo tempo, com exceção de uma delas, sendo este amostrador definido como o branco de campo. O branco de campo é preparado de forma similar aos demais amostradores, porém, é mantido fechado durante o período em que a amostragem transcorre, para não ter contato com o ar amostrado, e tem como finalidade identificar se houve algum tipo de contaminação durante os processos de transporte e amostragem. Nos experimentos de comparação de método, os amostradores foram fixados a uma distânica de cerca de 1 metro da entrada de ar do amostrador ativo utilizado como referência. Após transcorrido o tempo de amostragem preestabelecido (fim da amostragem), todos os amostradores foram tampados, ao mesmo tempo, e novamente embalados em saco de polietileno, para que pudessem ser transportados para o laboratório, onde foram armazenados refrigerados até o momento de análise. Estudos mostraram que amostras provenientes de análises de $\mathrm{NO}_{2}$ atmosférico por amostragem passiva utilizando TEA como solução absorvente puderam ser armazenadas por até 6 meses, sem diferença significativa na concentração obtida para $\mathrm{NO}_{2}$ em comparação com as amostras analisadas logo após a exposição. ${ }^{15}$

Para o procedimento de determinação do dióxido de nitrogênio fixado nos filtros, estes foram retirados dos amostradores e cada filtro foi transferido a um béquer contendo $2,5 \mathrm{~mL}$ de solução metanoica $5 \%(\mathrm{v} / \mathrm{v})$, para dessorção do analito retido no filtro de celulose. A solução metanoica é menos polar que a água pura e com isto facilita a solubilização da trietanolamina contendo o nitrito $\left(\mathrm{NO}_{2}{ }^{-}\right)$. O eluato é passado de forma quantitativa para um balão volumétrico de $5 \mathrm{~mL}$ e o volume do balão completado com o reagente de Griess-Saltzman. A solução resultante tem coloração rósea estabilizada após cerca de 15 minutos do início da reação. Para a determinação do teor do dióxido de nitrogênio no ar, divide-se a concentração encontrada de nitrito por 0,72 , uma vez que observações empíricas demonstraram que $0,72 \mathrm{~mol}$ de nitrito produz cor equivalente a $1 \mathrm{~mol}$ de $\mathrm{NO}_{2}{ }^{24,25}$

Para as medidas de absorbância da solução colorida, foi utilizado o espectrofotômetro Hitachi U-2000. As medidas de absorbância foram feitas com utilização de cubeta de caminho óptico de 1,0 cm em comprimento de onda de $540 \mathrm{~nm}$. A solução utilizada como branco foi a solução metanoica 5\% (v/v), o mesmo solvente utilizado nas extrações.

\section{RESULTADOS E DISCUSSÃO}

Para a determinação da concentração molar de $\mathrm{NO}_{2}$ coletado nos filtros impregnados foi necessária a utilização de uma curva analítica, previamente elaborada, que correlacionasse concentrações conhecidas de soluções de nitrito com medidas de absorbância em $540 \mathrm{~nm}$. Cada ponto da curva foi obtido de experimentos realizados em triplicata e o desvio padrão relativo médio apresentado foi de 9,72\%. A curva analítica obtida apresentou comportamento linear dentro da faixa de concentração de 2,50 a 20,0 $\mu$ mol $\mathrm{NO}_{2}{ }^{-} \mathrm{L}^{-1}$. A curva obtida pode ser expressa pela Equação 5:

$\mathrm{Abs}_{(540 \mathrm{~nm})}=3,94( \pm 0,02)\left[\mathrm{NO}_{2}^{-}\right] \times 10^{4}-4,00( \pm 0,20) \times 10^{-4}$
$\mathrm{R}^{2}=0,999$

em que $\left[\mathrm{NO}_{2}^{-}\right]$é a concentração do nitrito em mol L-1 e Abs o valor de absorbância da solução.

Inicialmente procedeu-se a validação e calibração do amostrador proposto. Estes experimentos foram feitos no mesmo local de instalação do amostrador de monitoramento contínuo para $\mathrm{NO}_{\mathrm{x}}$ (modelo $42 \mathrm{i}$ Thermo Environment), na Estação de Monitoramento da Qualidade do Ar da Companhia Ambiental do Estado de São Paulo (CETESB) na cidade de Araraquara.

Foram feitas amostragens em diferentes dias dos meses março, abril e maio do ano de 2016 com tempos de amostragem variando de 30 a 100 horas. Esse tempo foi estabelecido procurando otimizar o tempo de amostragem e as limitações do amostrador em medidas de campo. $\mathrm{O}$ mecanismo da reação de TEA com $\mathrm{NO}_{2}$ ainda não está bem estabelecido, mas a estequiometria proposta para $\mathrm{NO}_{2}$ em solução aquosa fornece uma relação molar de $2 \mathrm{NO}_{2}: 1 \mathrm{TEA}$ (Equação 6) ${ }^{26}$ Como foram utilizados $80 \mu \mathrm{L}$ da solução absorvente ( $11 \% \mathrm{v} / \mathrm{v}$ TEA, com densidade de $1,13 \mathrm{~g} \mathrm{~mL}^{-1}$ e $140 \mathrm{~g} \mathrm{~mol}^{-1}$ ), cada filtro impregnado contem cerca de $60 \mu \mathrm{mol}$ de TEA. Isto significa que, considerando-se eficiência de coleta de $100 \%$, seria possível reter cerca de $120 \mu \mathrm{mol}$ de $\mathrm{NO}_{2}$ ou cerca de $3000 \mu \mathrm{L}$ de $\mathrm{NO}_{2}$ a $25^{\circ} \mathrm{C}$. Concentrações atmosféricas para $\mathrm{NO}_{2}$ foram reportadas com valores médios de 21,0 $\pm 1,3 \mu \mathrm{g} \mathrm{m}^{-3}$ em área urbana ${ }^{16}$ e 53,02 $\pm 24,82 \mu \mathrm{g} \mathrm{m}^{-3}$ em região urbana com forte influência agrícola ${ }^{9}$ e com valores entre 
20,68 e 32,34 $\mu \mathrm{g} \mathrm{m}^{-3}$ em região litorânea industrializada ${ }^{27}$ e entre 0,32 à $17,13 \mu \mathrm{g} \mathrm{m}^{-3}$ em área montanhosa com influência de massas de ar provenientes de áreas urbanas. ${ }^{28}$

$2 \mathrm{NO}_{2(\mathrm{~g})}+\left(\mathrm{HOCH}_{2} \mathrm{CH}_{2}\right)_{3} \mathrm{~N}_{(\mathrm{aq})} \rightarrow\left(\mathrm{HOCH}_{2} \mathrm{CH}_{2}\right)_{3} \mathrm{NNONO}_{3 \text { (aq) }}$

$\left(\mathrm{HOCH}_{2} \mathrm{CH}_{2}\right)_{3} \mathrm{NNONO}_{3(\mathrm{aq})}+\mathrm{H}_{2} \mathrm{O} \rightarrow\left(\mathrm{HOCH}_{2} \mathrm{CH}_{2}\right)_{3} \mathrm{NHNO}_{3} \cdot \mathrm{HNO}_{2 \text { (aq }}$

Cada amostragem resultou em três medidas de concentração de $\mathrm{NO}_{2}$ e mais uma de branco de campo. Do valor médio do sinal das três absorbâncias, referentes às amostragens paralelas de $\mathrm{NO}_{2}$, foi descontada a absorbância do branco de campo e o valor obtido corresponde à concentração de $\mathrm{NO}_{2}$ para aquele período de amostragem. Foi utilizada a Equação 3 e a relação de $0,72 \mathrm{~mol}$ de nitrito para 1 mol de $\mathrm{NO}_{2}$ para se obter a concentração deste gás no ambiente $\left(\mathrm{C}_{\mathrm{x}}\right)$ e os resultados foram transformados em unidade de $\mu \mathrm{g} \mathrm{m}^{-3} \mathrm{de} \mathrm{NO}_{2}$. A Equação 3 fornece a concentração média referente ao tempo de amostragem e a quantidade de gás coletado $\left(\mathrm{n}_{\mathrm{x}}\right)$. Para conversão de $\mu \mathrm{g} \mathrm{m}^{-3}$ para ppbv pode ser utilizada a relação de $1 \mathrm{ppbv}-1,88 \mu \mathrm{g} \mathrm{m}^{-3} \mathrm{de} \mathrm{NO}_{2}$ $\left(25^{\circ} \mathrm{C}\right)$, baseada na massa molar desse composto.

Os dados de $\mathrm{NO}_{2}$ do amostrador ativo, utilizados como referência, são disponibilizados no portal da CETESB. O equipamento determina a concentração de $\mathrm{NO}$ e $\mathrm{NO}_{2}$ continuamente e a média das medidas de $\mathrm{NO}_{2}$ é fornecida de hora em hora. Para comparação entre os dois amostradores, foi utilizado o valor médio das concentrações horárias da CETESB correspondentes ao período de amostragem determinado para o amostrador passivo, situado no mesmo local de exposição. As concentrações referentes à essas amostragens encontram-se na Tabela 1. As diferenças de valores para a concentração de $\mathrm{NO}_{2} \mathrm{em}$ amostradores passivos em relação à amostradores ativos podem ser resultado de reações químicas que ocorrem dentro do caminho de difusão do amostrador passivo, como uma consequência do atraso entre a amostragem do ar ambiente desde a membrana porosa até a captura de $\mathrm{NO}_{2}$ pela superfície absorvente. ${ }^{29}$

Tabela 1. Concentrações de $\mathrm{NO}_{2}$ obtidas pelos métodos de amostragem passiva e ativa

\begin{tabular}{cccc}
\hline $\begin{array}{c}\text { Tempo de } \\
\text { amostragem (h) }\end{array}$ & $\begin{array}{c}\text { Concentra- } \\
\text { ção média e } \\
\text { desvio padrão } \\
\text { amostragem } \\
\text { passiva }\left(\mu \mathrm{g} \mathrm{m}^{-3}\right)\end{array}$ & $\begin{array}{c}\text { Concentra- } \\
\text { ção média } \\
\text { amostragem } \\
\text { ativa }\left(\mu \mathrm{g} \mathrm{m}^{-3}\right)\end{array}$ & $\begin{array}{c}\text { Desvio padrão } \\
\text { relativo entre } \\
\text { amostragem } \\
\text { passiva e } \\
\text { amostragem } \\
\text { ativa }(\%)\end{array}$ \\
\hline 29,9 & $15,7 \pm 2,8$ & 12,4 & 22,2 \\
32,3 & $18,3 \pm 0,8$ & 13,3 & 5,6 \\
42,4 & $24,8 \pm 2,2$ & 27,9 & 8,1 \\
51,5 & $28,1 \pm 4,3$ & 34,9 & 12,4 \\
77,5 & $18,7 \pm 1,8$ & 16,0 & 10,9 \\
100 & $22,4 \pm 0,7$ & 22,5 & 3,0 \\
\hline
\end{tabular}

Com os dados apresentados na Tabela 1 , foi possível estabelecer uma correlação entre as concentrações de $\mathrm{NO}_{2}$ encontradas pelos dois amostradores (Figura 3). A partir dessa correlação é possível corrigir as concentrações de $\mathrm{NO}_{2}$ obtidas pelo amostrador passivo confeccionado em relação ao amostrador de referência administrado pela CETESB, que por sua vez é calibrado com a frequência necessária para validar as medidas obtidas e necessárias para o acompanhamento da qualidade de ar da cidade de Araraquara. A curva fornecida por essa correlação é representada pela Equação 7:

$\left[\mathrm{NO}_{2}\right]_{\text {CORRIGIDO }}=1,91( \pm 0,14)\left[\mathrm{NO}_{2}\right]_{\text {PASSIVO }}-19,7( \pm 3,1)$

$\mathrm{R}^{2}=0,979$ em que $\left[\mathrm{NO}_{2}\right]_{\text {CORRIGIDO }}$ é a concentração de $\mathrm{NO}_{2}$ em $\mu \mathrm{g} \mathrm{m}^{-3}$ corrigida com relação à concentração de $\mathrm{NO}_{2}$ do amostrador de referência. Os resultados obtidos permitem que valores de concentração encontrados com o uso dos amostradores passivos confeccionados sejam corrigidos por meio de um valor de referência.

A US National Institute Of Safety and Health (NIOSH) e o Parlamento Europeu e Conselho da União Européia (Comitê Europeu - CE) estabelecem que se os desvios obtidos em medidas realizadas no ambiente de trabalho e em ambientes externos com o uso de amostrador passivo estiverem dentro de uma faixa de $\pm 25 \%$ os valores medidos podem ser considerados estatisticamente significativos. ${ }^{30,31}$ Para avaliar se os dados obtidos neste experimento estão dentro desta faixa, foram apresentadas, na Figura 3, duas retas construídas sobre os limites de $25 \%$, determinados a partir da linha de tendência das medidas. A linha de tendência é usada como referência, pois representa a linearização dos pontos referentes às medidas de $\mathrm{NO}_{2}$, obtidas pelo amostrador ativo e pelo amostrador passivo.

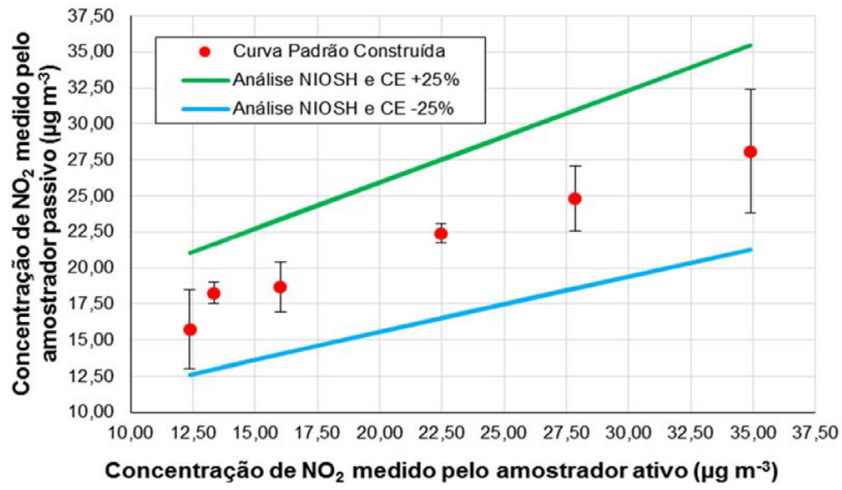

Figura 3. Correlação entre os resultados obtidos pelo amostrador passivo e o amostrador ativo administrado pela CETESB (SP), com os limites superiores e inferiores considerando-se desvios de $\pm 25 \%$ estabelecidos pelas agências regulatórias para uso de amostradores passivos

Os resultados dos limites, medidos a partir do resultado determinado pelo sinal analítico, mostram que todos os dados experimentais obtidos com o amostrador passivo estão dentro dos limites de $\pm 25 \%$ permitidos, portanto, o amostrador passivo proposto pode ser utilizado em medidas de ambiente de trabalho segundo as normas da NIOSH e do CE.

\section{CONCLUSÃO}

As concentrações de $\mathrm{NO}_{2}$, em $\mu \mathrm{g} \mathrm{m}^{-3}$, determinadas pelo amostrador confeccionado e o amostrador ativo gerenciado pela CETESB, apresentam todos os pontos da curva de concentração corrigida dentro de uma faixa de erro de $25 \%$, o que, segundo a NIOSH e o CE, permite concluir que o amostrador construído é eficaz e satisfatório para determinação da concentração de $\mathrm{NO}_{2}$ no ar em ambientes externos. Além disso, o amostrador confeccionado possui dimensões que resultam em uma relação de área da seção transversal sobre o comprimento de caminho de difusão que possibilita maior quantidade de gás coletado e permite realizar as amostragens em tempos menores do que seria necessário com o uso de amostradores convencionais para coletar a mesma quantidade de gás. Mesmo com a utilização de materiais de baixo custo facilmente encontrados no mercado nacional foi possível preparar um amostrador passivo e validar suas condições de uso. Com isto, é possível que sua utilização seja ampliada para que possam ser empregados na solução de problemas envolvendo a determinação de $\mathrm{NO}_{2}$ em diversos ambientes, mesmo que para posterior análise estejam disponíveis apenas um espectrofotômetro simples e materiais básicos de laboratório. 


\section{MATERIAL SUPLEMENTAR}

Fotos do amostrador e do suporte utilizado estão disponíveis em http://quimicanova.sbq.org.br, na forma de arquivo PDF, com acesso livre.

\section{AGRADECIMENTOS}

P. A. F. Souza, K. C. A. Francisco e A. A. Cardoso agradecem à CETESB e seus funcionários, Maria Lucia Gonçalves Guardani e Jorge Luís Carizia, pela disponibilização do espaço físico e dos dados utilizados nesse trabalho. Os autores agradecem ainda ao $\mathrm{CNPq}$ e à CAPES pelas bolsas concedidas e pelo auxílio financeiro para desenvolvimento do projeto.

\section{REFERÊNCIAS}

1. WHO Regional Office for Europe; Air Quality Guidelines, Global Update 2005, World Health Organisation: Copenhagen, 2006.

2. Garcia, G.; Allen, A. G.; Cardoso, A. A.; Water Air Soil Pollut. 2014, 225,1836

3. Campos, V. P.; Cruz, L. P. S.; Alves, E. M.; Santos, T. de S.; Silva, A. D.; dos Santos, A. C. C.; Lima, A. M. V.; Paixão, C. S.; Santos, D. C. M. B.; Brandão, D. S.; Andrade, E. J. S.; Moreira Jr., J. I.; Conceição, K. C. S.; Ramos, M. de S.; Pontes, M. C. G.; do Amaral, M. F.; Mattos, R. R.; Quim. Nova 2006, 29, 872.

4. Garcia, G.; Allen, A. G.; Cardoso, A. A.; J. Environ. Monit. 2010, 12, 1325.

5. Finlayson-Pitts, B. J. F.; Pitts Jr., J. N.; Atmospheric Chemistry Fundaments and Experimental Techniques, John Wiley \& Sons: New York, 1986.

6. Tang, Y. S.; Cape, J. N.; Sutton, M. A.; The Scientific World 2001, 1, 513.

7. Arbex, M. A.; Martins, L. C.; Pereira, L. A. A.; Negrini, F.; Cardoso, A. A.; Melchert, W. R.; Arbex, R. F.; Saldiva, P. H. N.; Zanobetti, A.; Braga; A. L. F.; Braz. J. Med. Biol. Res. 2007, 40, 527.

8. Ugucione, C.; Machado, C. M. D., Cardoso, A. A.; Quim. Nova 2009, $32,1829$.
9. Machado, C. M. D., Cardoso, A. A., Allen, A. G.; Environ. Sci. Technol. 2008, 42,381

10. Allen, A. G.; Cardoso, A. A.; Wiatr, A. G.; Machado, C. M. D.; Paterlini, W. C.; Baker, J.; J. Braz. Chem. Soc. 2010, 21, 87.

11. Allen, A. G., Machado, C. M. D., Cardoso, A. A.; Environ. Pollut. 2011, 159,1190 .

12. Palmes, E. D.; Gunnison, A. F.; DiMattio, J.; Tomczyk, C.; Am. Ind. Hyg. Assoc. J. 1976, 37, 570.

13. Ankersmit, H. A.; Tennet, N. H.; Watts, S. F.; Atmos. Environ. 2005, 39, 695.

14. Melchert, W. R.; Cardoso, A. A.; Quim. Nova 2006, 29, 365.

15. Krochmal, D.; Górski, L.; Environ. Sci. Technol. 1991, 25, 531.

16. Campos, V. P.; Cruz, L. P. S.; Godoi, R. H. M.; Godoi, A. F. L.; Tavares, T. M.; Microchem. J. 2010, 96, 132.

17. Cox, R. M.; Environ. Pollut. 2003, 126, 301.

18. Sekine, Y.; Watts, S. F.; Rendell, A.; Butsugan, M.; Atmos. Environ. 2008, 42, 4079 .

19. Pitombo, L. R. M.; Cardoso, A. A.; Int. J. Environ. Anal. Chem. 1990, 39, 349 .

20. Massman, J. W.; Atmos. Environ. 1998, 32, 1111.

21. Harper, M.; Purnell, C. J.; Am. Ind. Hyg. Assoc. J. 1987, 48, 214.

22. Heal, M. R.; Cape, J. N.; Atmos. Environ. 1997, 31, 1911.

23. Yu, Chang Ho; Morandi, M. T, Weisel, C. P.; J. Expo. Sci. Environ. Epidemiol. 2008, 18, 441.

24. Lodge Jr, J. P.; Methods of air sampling and analysis, $3^{\text {rd }}$ ed., Michigan, 1998.

25. Shaw, J. T.; Atmos. Environ. 1967, 1, 81.

26. Gold, A.; Anal. Chem. 1977. 49, 1448.

27. Allen, A. G.; McGonigle, A. J. S.; Cardoso, A. A.; Machado, C. M. D.; Davison, B.; Paterlini, W. C.; da Rocha, G. O.; de Andrade, J. B.; J. Braz. Chem. Soc. 2009, 20, 214.

28. Gao, X; Xue, L; Wang, X; Wang, T; Yuan, C; Gao, R; Zhou, Y; Nie, W; Zhang, Q; Wang, W.; Sci. Tot. Environ. 2012, 433, 498.

29. Heal, M. R.; O'Donoghue, M. A.; Cape, J. N.; Atmos. Environ. 1999, 33,513 .

30. Seethpathy, S.; Górecki, T.; Li, X.; J. Chromatogr. A 2008, 1184, 234.

31. ht t p://eur-1ex.europa.eu/legal-content/PT/TXT/ PDF/?uri=CELEX:32008L0050\&from=PT, acessada em agosto 2017. 